\title{
Les Determinants Demographiques Et Socio-Economiques Du Confiage Des Enfants Au Burkina Faso
}

\author{
Zourkaléini Younoussi \\ Université de Ouagadougou \\ Institut Supérieur des Sciences de la Population (ISSP) \\ 03 BP 7118 Ouagadougou 03
}

Tél. : (226) 50302558 / 50302559

Fax : (226) 50302560

E.mail :yzourkaleini@issp.bfRésumé

\begin{abstract}
RESUME
Au Burkina Faso, comme dans plusieurs autres pays de l'Afrique sub-saharienne, traditionnellement, nombreux sont les enfants qui par la pratique du confiage sont temporairement ou dans le long terme placés par leurs parents biologiques dans d'autres familles (le plus souvent apparentées) avec pour objectifs leur socialisation et leur éducation. La présente étude montre d'abord, qu'avec la crise économique la pratique de confiage s'est amplifiée ; ensuite, elle n'échappe pas à l'influence des inégalités sociales et économiques entre les familles, des groupes sociaux et des milieux de résidence et enfin on observe un changement dans le temps de ses déterminants. La reproduction de cette pratique dans un contexte social, économique et géographique différents favorise de nouvelles formes et de modalités qui donnent lieu à penser à l'exploitation et au trafic des enfants.
\end{abstract}




\section{Introduction}

Le confiage, encore appelé placement ou encore transfert des enfants, constitue un des traits traditionnels des systèmes familiaux africains qui se pratique sur la base du respect de certaines règles (Jonkers 1997). Le concept «enfant confié » ou « enfant placé » ou encore " enfant transféré » se rapporte à une forme d'organisation sociale qui intègre l'enfant dans un système d'échange entre familles avec pour objet sa socialisation et son éducation. C'est le type de confiage jadis pratiqué en Afrique de l'Ouest et qui se distingue de l'adoption (concession définitive). Par ce système d'échange, l'enfant est placé ou transféré auprès d'un parent, d'un ami, d'un maître coranique ou d'une simple connaissance pour être élevé comme les enfants de ce dernier.

Par cette pratique l'enfant se retrouve dans un processus de formation tout en contribuant souvent à l'activité économique du ménage dont il est membre. Quand la contribution à l'activité économique devient le seul objet du confiage au détriment du processus de formation l'enfant peut être exploité. Dans la perspective que l'économique prend le dessus sur le social, plusieurs auteurs s'accordent à dire que, pour les parents directs, le placement est un moyen de se «délester » d'une partie de la " charge » de leurs descendants, lorsqu'ils en ont un grand nombre ou qu'ils sont dans l'incapacité de leur assurer le minimum vital (Locoh 1995b; Frank 1985). Dans un tel contexte la pratique du confiage des enfants peut perdre son fondement traditionnel pour s'inscrire dans une logique de transfert des enfants pour des raisons économiques. On parle aujourd'hui des dérapages qui conduisent à un nouveau visage de ce fait social sur lequel émerge le scandale du trafic des enfants.

Au Burkina Faso, on déplore aujourd'hui le fait que la paupérisation croissante de la population et les contraintes économiques rendent plus difficile l'accueil traditionnel. Cette pauvreté aidant, on peut donc s'attendre à un ralentissement de cette pratique, les capacités des familles plus «riches" susceptibles d'accueillir les enfants connaissant certaines limites. Cependant, sous le poids de la pression sociale, une famille se sent parfois obligée d'accueillir un enfant qui souvent, représente une charge supplémentaire et qui ne peut donc pas bénéficier des soins les plus élémentaires pour son épanouissement. Au lieu d'être donc protégé ou inséré, cet enfant confié peut se voir rejeté et exclu, voire exploité. Si en tant de crise le nombre d'enfants confiés 
Zourkaléini Younoussi: Les Determinants Demographiques Et Socio-Economiques Du Confiage Des Enfants Au Burkina Faso

augmente c'est au niveau de la qualité et de la nature qu'il faille rechercher les changements.

A partir des données de l'enquête nationale sur «les migrations, l'insertion urbaine et l'environnement au Burkina Faso $2000 »$, cet article poursuit les objectifs suivants: examiner la tendance du confiage des enfants de moins de 14 ans au Burkina Faso, mettre en évidence les facteurs démographiques, socioéconomiques et culturels qui déterminent ce confiage et, enfin, de déterminer les éventuels changements intervenus au cours du temps dans le profil des déterminants.

Deux outils essentiels de l'analyse des biographies, les courbes de survie de Kaplan Meier et les régressions semiparamétriques à risques proportionnels, sont utilisés pour évaluer les probabilités d'accéder au confiage ou pour mieux comprendre ce qui mène au confiage. L'examen des déterminants du confiage par génération nous permet de mettre en évidence les changements intervenus en passant de la génération la plus vieille à la plus jeune.

\section{I) Le confiage des enfants : une pratique traditionnelle}

La pratique de confiage, de placement ou de transfert des enfants s'appui sur les coutumes et us qui sont activées par les besoins qui se manifestent au niveau familial ou individuel (voir schéma 1). En Afrique de l'Ouest, traditionnellement l'enfant n'est pas la propriété de ses parents, il appartient à toute la famille, à toute la communauté. Chez ses géniteurs ou ailleurs, dans un autre cercle familial, il doit pouvoir se sentir à la "maison». Chaque parent doit non seulement pouvoir élever, garder et éduquer ses enfants mais aussi en recevoir d'autres. Ainsi, les parents remettent volontiers les enfants aux soins de proches. On soutient souvent l'idée qu'il faut laisser l'éducation de l'enfant à d'autres parents car loin des parents géniteurs, il sera plus docile et plus réceptif aux bonnes méthodes éducatives (Fagnon et Kpadonou 1997). Les ménages d'accueil sont entièrement responsables pendant les années de garde sans que pour autant les parents donateurs se soustraient à leurs devoirs ou perdent leurs droits.

Le confiage a une durée limitée et ne débouche pas sur l'adoption de type occidental. Contrairement à l'idée admise en Occident, le confiage d'enfants ne sert que rarement à adopter des 
orphelins ou à donner des enfants aux couples stériles. La mobilité enfantine n'est jamais automatique, il faut qu'on ait envie ou besoin de cette présence enfantine pour la demander ou l'accepter (Jonckers 1997). Mais, cette interaction entre famille ne fait jamais appel à une institution de type formel et passe souvent par une convention sociale.

Le confiage peut avoir lieu dans des sociétés et des milieux très différents. En dépit des différences entre leur niveau de vie, les citadins et leurs parents ruraux peuvent se confier mutuellement des enfants. Les différents types de confiage relèvent avant tout de la volonté de resserrer les relations de parenté et d'alliance (Lallemand 1993; Jonckers 1994). Il faut reconnaître que dans la société traditionnelle d'Afrique de l'Ouest et du Burkina Faso en particulier, le confiage des enfants en milieu rural à une famille apparentée ou amie revêt une dimension éducative et sociale. La dimension éducative découle du fait qu'en milieu rural, l'enfant élevé ailleurs que dans son foyer naturel est traité avec moins d'indulgence par des tuteurs garants des traditions et valeurs sociales. En milieu urbain, le confiage permet à l'enfant issu d'un milieu rural ou pauvre d'être scolarisé ou d'apprendre un métier.

Le confiage se pratique aussi dans l'intérêt des parents d'accueil et de départ. Les premiers tirent un bénéfice en termes de compensation matrimoniale (un enfant cédé en échange de sa mère), d'expression ou de réception de solidarité familiale avec les autres membres de leurs familles et dans une moindre mesure s'accaparer des domestiques (Lallemand 1994 ; Locoh 1988). Aussi, en milieu rural comme en milieu urbain, bien que l'enfant accueilli effectue, comme chez lui, des petits services pour compenser sa prise en charge, cet aspect économique ne devrait pas prendre le pas sur les motivations premières de son transfert.

Quant aux familles de départ, on évoque souvent comme raison le déséquilibre entre les ressources disponibles et les besoins réels de la famille à satisfaire, qui parfois est caché derrière le désir ou l'obligation de faire plaisir, amène des parents à solliciter ou à accepter le partage de la garde des enfants (Lloyd et Blanc 1996; Locoh 1995b). Dans les cas extrêmes le désir de protéger les enfants contre les sorciers et les jeteurs de mauvais sort est aussi une autre raison avancée (Fagnon et Kpadonou 1997). 
Zourkaléini Younoussi: Les Determinants Demographiques Et Socio-Economiques Du Confiage Des Enfants Au Burkina Faso

Un enfant peut être placé auprès de ses grands parents en remplacement de sa mère. Plus généralement, pour certaines familles où le développement économique et social n'a pas eu pour conséquence la nucléarisation des familles, on peut s'attendre à ce que les personnes âgées élèvent les enfants et organisent leur confiage entre différents parents ou alliés. Ainsi les enfants dont les grands-parents sont encore en vie peuvent être plus exposés au confiage.

Plus que les garçons les filles peuvent se voir confier la garde des nourrissons ou des travaux domestiques. Ainsi, le sexe de l'enfant peut être un élément qui intervient au moment de la concertation entre les deux familles (accueil et de départ). Aux besoins, des familles d'accueil, on peut s'attendre que les filles soient plus confiées que les garçons (Jonckers 1997).

Dans plusieurs sociétés ouest africaines et en particulier au Burkina Faso, les échanges d'enfants peuvent revêtir une haute valeur symbolique. On peut donc s'attendre à ce que des frères et sœurs se confient mutuellement la garde de leurs enfants et qu'une mère accepte de céder son jeune enfant dès qu'elle en a un nouveau né. En outre, on peut s'attendre à ce que l'enfant sevré suite à la grossesse de la mère soit placé auprès d'une tante ou de sa grand-mère. Le caractère symbolique du placement d'enfants peut apparaître également lorsqu'on donne un enfant à un jeune couple pour favoriser l'arrivée de sa descendance. Il en va de même lorsqu'on confie un bébé pour déjouer le destin (si sa mère a perdu beaucoup d'enfants avant lui) ou le protéger (si l'on considère qu'il est menacé par des forces occultes néfastes). Par ailleurs, celui ou celle qui garde un bon souvenir de sa tutrice ou de son tuteur lui confira son propre enfant. Réciproquement, un enfant confié peut, devenu adulte, élever des descendants de son ancien tuteur. Les transferts et les placements pour des raisons occultes peuvent ausi s'expliquer par l'origine ethnique ou l'éducation qui sont en interaction avec les croyances et valeurs en vigueur dans la société.

Le confiage, comme une des manifestations des solidarités familiales, reste une pratique courante, mais des changements semblent s'opérer. Aujourd'hui avec la crise qui affecte les familles, les solidarités traditionnelles, extraordinairement opérantes jusqu'à récemment, semblent atteindre leurs limites. Avec la crise, le confiage comme pratique permettant une 
réallocation des charges de la reproduction démographique à l'intérieur de la famille, se trouve remis en cause car les capacités d'accueil des familles, en zone rurale comme en milieu urbain, sont considérablement affectées. Si en temps de crise le nombre d'enfants confiés augmente c'est au niveau de la qualité et de la nature qu'il faille rechercher les changements. De nos jours, dans un contexte économique marqué par la paupérisation des ménages, le travail des enfants confiés est de moins en moins un surplus pour devenir un minimum de survie dans la composition du revenu des ménages (Marcoux, 1997). D'où une diversification des tâches accomplies par l'enfant qui ne correspondent plus nécessairement à sa force de travail et à son âge mais, qui se font au détriment de son épanouissement et surtout de son éducation. Ce bouleversement est surtout perceptible en ville. Les jeunes ruraux confiés aux familles citadines dans le but d'être scolarisés ou d'accéder à l'apprentissage d'un métier peuvent malheureusement être confrontés à l'exploitation. Les filles peuvent effectuer des tâches domestiques et les garçons peuvent participer aux charges de la famille d'accueil en exerçant un petit métier dans le «secteur informel» de l'économie urbaine. Ils peuvent rejoindre donc le nombre croissant d'enfants exerçant un travail «précoce»: cireurs de chaussures, vendeurs de gâteaux ou de sachets d'eau.

Théoriquement, la ville offre un cadre socio-économique plus adapté à préparer l'enfant pour une plus longue scolarité ou pour l'apprentissage d'un bon métier. En effet, au Burkina Faso comme dans les autres pays d'Afrique subsaharienne où les structures scolaires et économiques sont plus développées en ville, en l'absence de crise économique, pour assurer une meilleure scolarité et partant, une bonne socialisation à leurs enfants, les familles vivant en milieu rural, souvent dépourvu de structures socio-économiques, devraient avoir plus de propension à confier leurs enfants aux membres de leur famille vivant en ville. Mais en temps de crise, l'expression de la solidarité familiale par le placement d'enfants confiés, si elle existe toujours dans sa forme traditionnelle, devrait inverser de sens. La crise socio-économique crée des conditions socio-économiques beaucoup plus difficiles en ville qu'au village. Cette situation singulière engendrée par le manque d'emploi rémunéré, pour certains parents, le salaire insuffisant et non disponible régulièrement pour d'autres, renforcé par les coûts de scolarisation et d'apprentissage très élevés, devrait pousser certains parents à envoyer leurs enfants au village où le coût de la vie en général et les frais de scolarité en particulier sont moins élevés. Par contre, si en temps de crise les flux sont toujours 
Zourkaléini Younoussi: Les Determinants Demographiques Et Socio-Economiques Du Confiage Des Enfants Au Burkina Faso

en direction des villes sans fléchissement ni même une augmentation en direction des villages ou encore une diminution $\mathrm{du}$ confiage dans son ensemble, alors on peut croire que les enfants sont plutôt victimes de formes subtiles du confiage qui les soumettent à toute forme exploitation et en ville particulièrement.

Quelle signification faut-il donner à la pratique actuelle du confiage des enfants au Burkina Faso? Cette pratique répond-elle toujours aux normes d'expression de solidarité familiale ou bien est-elle en train de prendre la forme d'exploitation des enfants? Il s'agira pour nous de mettre en évidence les facteurs démographiques, socio-économiques et culturels qui déterminent ce confiage et, enfin, de déterminer les éventuels changements intervenus au cours du temps dans le profil des déterminants. Ces changements seront mis en évidence par un examen des déterminants du confiage par génération. S'agissant des facteurs explicatifs nous supposons que: les premiers enfants et plus particulièrement les filles, plus susceptibles de remplacer leur mère, ont une probabilité plus élevée d'être confiés; les enfants qui ont plus d'oncles et/ou de tantes ont une probabilité plus élevée d'être placés pour tenir compte du fait que des frères et sœurs se confient mutuellement la garde de leurs enfants; l'origine ethnique a un impact sur la circulation des enfants; certaines pratiques chez les musulmans exposent plus leurs enfants au placement; l'éducation des parents est négativement associée au confiage des enfants alors que la résidence en milieu rural lui est positivement associée. 
Schéma 1 : Cadre conceptuel du confiage traditionnel des enfants

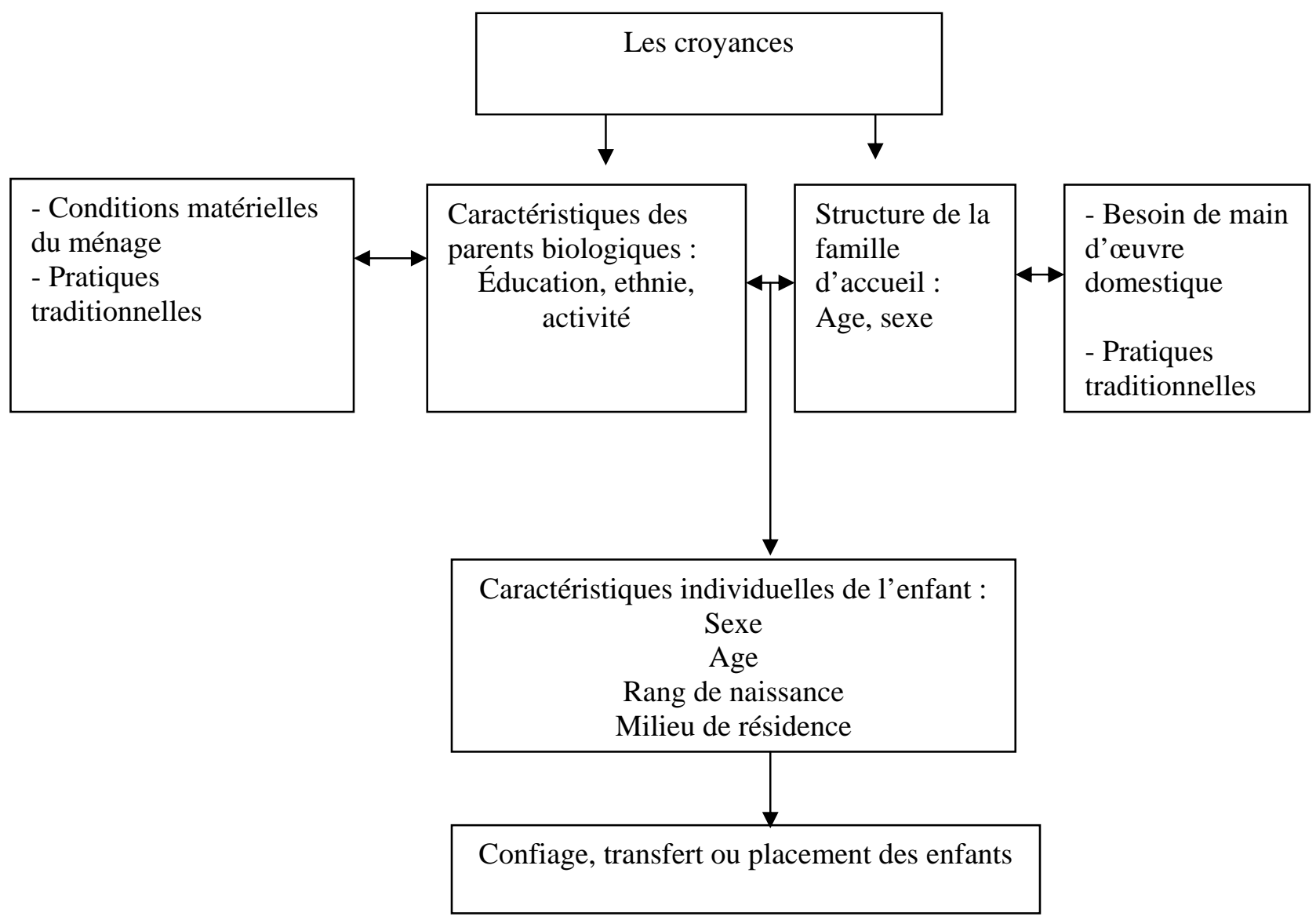


Zourkaléini Younoussi: Les Determinants Demographiques Et Socio-Economiques Du Confiage Des Enfants Au Burkina Faso

\section{II) Données et méthodes d'analyse}

Les données utilisées dans cette étude proviennent de l'enquête nationale réalisée en 2000 sur « les migrations, l'insertion urbaine et l'environnement au Burkina Faso » et qui porte sur 8644 biographies migratoires individuelles. Parmi celles-ci, on compte 4568 biographies de femmes d'âge compris entre 15 et 64 ans dont 3749 ont déclaré 17540 naissances survenues entre 1952 et 2000. Le module « histoire génésique et devenir des enfants », du questionnaire biographique-femme, a permis de recueillir auprès de chaque femme, le premier départ éventuel de chacun de ses enfants, l'âge auquel ce départ a eu lieu, le motif de départ, le département et la province (ou le pays) de destination.

L'analyse est centrée sur le premier départ, sous l'hypothèse que le devenir de l'enfant reste fortement influencé par son premier départ. Nous voulons en savoir plus sur les facteurs démographiques, socio-économiques et culturels qui favorisent ce départ.

Les migrations économiques, scolaires (scolarisation dans le second cycle), de mariages ou d'apprentissage interviennent généralement à des âges relativement élevés et peuvent généralement s'inscrire dans le cadre d'alliance, d'entraide familiale de type économique ou de poursuite d'études qui ne correspondent pas souvent à un confiage de type traditionnel. Elles relèvent plus d'une contrainte que d'un acte volontaire s'inscrivant dans le processus de socialisation de l'enfant. L'enfant est souvent partie prenante des conditions de son départ et souvent le principal investigateur. Dans le cas des départs en bas âge, ils ne peuvent avoir lieu qu'après le consentement des deux familles (départ et accueil). Ces enfants seront donc considérés comme des enfants transférés, placés ou confiés, lorsque le parent rejoint, avant 14 ans, n'est pas le père biologique (puisqu'ils quittent leurs mères) ou l'époux/l'épouse (quel que soit l'âge au départ). Bien que cette limite d'âge reste arbitraire, à partir de 14 ans, l'enfant n'est plus considéré comme étant exposé au risque de confiage traditionnel puisque l'absence d'un collège d'enseignement général dans la localité pourra être le mobile principal du déplacement. Cette limite d'âge permet donc d'exclure les enfants qui sont contraints de quitter le village après la fin du cycle primaire ou qui sont économiquement actifs à partir de 14 ans. 
Les lieux de départ et d'arrivée des enfants sont tous ceux que leur mère a connus au moment où ils avaient moins de 14 ans. En cas de confiage le lieu d'arrivée est la destination de l'enfant et le lieu de départ est celui où il résidait avec sa mère avant son départ, qu'il soit à l'intérieur ou à l'extérieur du pays (si la mère avait séjourné avant de se retrouver au Burkina). En l'absence de déplacement de la mère (jusqu'à ce que l'enfant atteigne 13 ans) et de l'enfant les lieux de départ et d'arrivée sont assimilés au lieu de résidence de la mère au moment où l'enfant avait moins de 14 ans.

On remarquera que l'absence d'information sur les enfants dont les mères n'étaient pas en vie au moment de l'enquête constitue une des limites de nos données. Par ailleurs, si nos données ne permettent pas de prendre en compte les enfants dont les mères sont décédées, INSD et ORC Macro (2004) montrent que ceux-ci ne peuvent que représenter que moins de $2 \%$ de l'ensemble des enfants de moins de 15 ans vivant dans un ménage en l'absence de leur mère et père. Ce pourcentage pouvant être encore plus faible si nous excluons les enfants âgés de 14, pour tenir compte de la tranche d'âge que nous avons retenons dans le cadre de cette étude.

Compte tenu du faible niveau de l'espérance de vie au Burkina Faso (48 ans), les femmes très âgées sont faiblement représentées dans l'échantillon. Leurs enfants ne sauraient donc être représentatifs des enfants dont les mères auraient survécu à ces groupes d'âge. Pour minimiser ce biais, nous excluons de l'analyse tous les enfants de mères âgées de plus de 60 ans $^{1}$ au moment de l'enquête. Ainsi notre étude porte sur un nombre total de 17121 enfants (nés entre 1955 et 1999) de 3692 femmes.

Les activités économiques de la mère ou ses niveaux d'éducation atteints sont toutes celles exercées ou ceux acquis avant le quatorzième anniversaire de son enfant. Les enfants d'une même mère peuvent donc ne pas avoir les mêmes lieux de départ ou d'arrivée; la mère peut ne pas exercer le même type d'activité au moment où chacun de ses enfants avait moins de 14 ans.

Une analyse descriptive nous permet de prendre connaissances des parents d'accueil, des lieux de départ et de destination. Les courbes de survie de Kaplan Meier nous permettent de mettre en évidence l'évolution du confiage dans le

\footnotetext{
${ }^{1}$ Le choix de cet age se justifie par la chute brutale des effectifs des femmes après cet age

212
} 
Zourkaléini Younoussi: Les Determinants Demographiques Et Socio-Economiques Du Confiage Des Enfants Au Burkina Faso

temps ou la comparaison entre groupes. Afin d'évaluer l'influence exercée par chaque facteur sur la probabilité d'être confié nous utilisons un modèle de régression de survie. L'âge de l'enfant est utilisé comme variable mesurant le temps. Chaque enfant est suivi jusqu'à son départ et en l'absence de départ l'enfant est censuré à l'âge atteint ou après 13 ans lorsqu'il est âgé de plus 13 ans au moment de l'enquête.

Cette analyse qui fait appel au modèle semi-paramétrique à risques proportionnels, utilise les variables explicatives relatives aux caractéristiques de l'enfant (sexe, rang de naissance, milieu de résidence au départ, lieu de destination, nombre d'oncles et/ou de tantes encore en vie, génération) et celles de la mère (religion, activité économique, niveau d'éducation et l'ethnie).

Afin d'examiner l'évolution, dans le temps, de la structure des déterminants du confiage, la génération est considérée comme groupe cible en maintenant les mêmes variables explicatives. Ainsi, au total nous présentons quatre modèles: un modèle sur l'ensemble de la période (1955-1999) et un modèle pour chacune des trois générations (enfants nés avant 1980, enfants nés entre 1980 et 1989 et enfants nés entre 1990 et 1999).

Les enfants dont les mères ont connu plusieurs déplacements ou plusieurs activités au moment où ils avaient moins de 14 ans se retrouvent dans plusieurs enregistrements de la base de données. Pour remédier à ce problème, les erreurs standards des coefficients de régression ont été ajustées en utilisant les erreurs standards de Huber-White (Hox, 2002).

Précisons que comme les données de toute enquête rétrospective, les données utilisées ici présentent un certain nombre de limites. En effet, la technique des biographies nécessite l'enregistrement chronologique de tous les événements survenus dans la vie de l'individu. Elle fait donc appel à la mémoire qui peut être défaillante sur certaines dates ou certains évènements. Toutefois, l'utilisation de la fiche "âge-évenement», qui a fait ses preuves dans ce genre d'enquête biographique permet de minimiser les biais de mémoire (Antoine et Piché, 1998). Malgré ces limites inhérentes à ce genre d'enquête, la base de données constitue une source unique et originale pour l'étude des facteurs associés à la circulation des enfants. L'enregistrement des biographies résidentielles et professionnelles des parents et de l'âge au départ et de l'origine et la destination de l'enfant fait la 
différence avec d'autres enquêtes dont les Enquêtes Démographiques et de Santé.

III) Quelques caractéristiques du confiage

Les filles représentent $48,5 \%$ des enfants confiés avant 14 ans. Les enfants confiés représentent $5 \%$ de la population totale féminine ou masculine de moins de 14 ans. Ainsi, dans l'ensemble, $5 \%$ des enfants ont connu une situation de transfert, de placement ou de confiage avant 14 ans. Ce résultat ne s'écarte pas trop de celui enregistré lors de l'Enquête Démographique et de Santé (EDS) réalisée en 2003 : 7,2\% des enfants de moins de 15 ans ne vivaient avec aucun parent alors que leurs mères sont encore en vie (INSD et ORC Macro 2004).

\section{A) Parents d'accueil des enfants confiés}

Indépendamment de l'âge au départ, le tableau 1 révèle que les enfants (filles comme garçons), sont essentiellement placés auprès des oncles/tantes, des grands-parents et des frères/sœurs. Entre 0 et 5 ans, les garçons sont plus accueillis par les grands parents (maternels comme paternels) alors que les filles sont confiées auprès des grands parents maternels. Les grands-parents accueillent près de $54 \%$ et $42 \%$ respectivement des garçons et des filles de 0 à 5 ans. Ces proportions tombent à $11 \%$ et $20 \%$ lorsque l'âge du petit garçon et/ou de la petite fille est compris entre 6 et 13 ans. Cette forme d'accueil des enfants par les grands-parents suppose que ces derniers participent encore à la gestion, à l'éducation et à l'élevage de leurs petits enfants. Ce phénomène est plus accentué lorsqu'il s'agit de l'accueil d'une fille par ses grands parents maternels (un tiers des filles confiées avant 6 ans), ce qui suggère que les femmes se laissent dessaisir de leurs filles à un âge précoce pour que celles-ci les remplacent auprès de leur mère.

Au delà de 5 ans aussi bien pour les filles que pour les garçons le confiage semble se réaliser plus auprès des oncles/tantes, en particulier les oncles/tantes paternels $(50 \%$ pour les garçons et $44 \%$ pour les filles). On retrouve donc une logique des obligations traditionnelles et sociales. Pour les oncles/tantes qui accueillent un neveu, une nièce, c'est l'occasion de constituer un capital de reconnaissance de la part de l'enfant du frère et/ou de la sœur, mais aussi de manifester son appui pour la prise en charge de l'enfant à eux tous (Locoh, 1995b). 
Zourkaléini Younoussi: Les Determinants Demographiques Et Socio-Economiques Du Confiage Des Enfants Au Burkina Faso

Malgré la jeunesse des enfants confiés, on constate que les frères et sœurs accueillent une part non négligeable. En effet, la descendance d'une femme étant généralement nombreuse, les enfants d'une même mère naissent souvent sur une période très étendue (il peut arriver que 20 ans séparent l'aîné du benjamin), et les règles impérieuses de solidarité entre germains d'une même mère sont toujours en vigueur. Les aînés accueillent et participent à l'éducation voire la scolarisation des cadets. Les garçons le sont plus avant 6 ans (13\%) alors que les filles sont beaucoup plus concernées après 5 ans (17\%).

Tableau 1 : Répartition $(\%)^{*}$ des enfants confiés par le parent rejoint selon l'âge au départ et le sexe

\begin{tabular}{|l|lll|lll|}
\hline & \multicolumn{5}{|c|}{ Age au départ } \\
Parent d'accueil & $0-5$ & & & $6-13$ & & \\
Lien de parenté avec & ans & & & & & \\
le parent rejoint & garçon & fille & Total & garçon & Fille & Total \\
Oncle/tante maternel & 7,7 & 17,6 & 13,6 & 6,5 & 15,7 & 10,4 \\
Oncle/tante paternel & 10,5 & 26,8 & 20,2 & 43,7 & 28,3 & 37,1 \\
Grand-parent maternel & 28,3 & 34,8 & 32,1 & 3,2 & 12,5 & 7,3 \\
Grand-parent paternel & 25,9 & 7,4 & 14,9 & 7,9 & 7,4 & 7,5 \\
Autre parent maternel & 2,7 & 5,7 & 4,5 & 3,9 & 5,3 & 4,5 \\
Autre parent paternel & 9,1 & 2,2 & 5,0 & 2,8 & 2,0 & 2,5 \\
Frère/sœur & 12,6 & 2,0 & 6,3 & 7,5 & 16,7 & 11,4 \\
Autre parent & 0,3 & 1,5 & 1,0 & 13,5 & 3,6 & 9,3 \\
Sans lien & 2,9 & 2,2 & 2,5 & 10,9 & 8,5 & 9,9 \\
Total & 100,0 & 100,0 & 100,0 & 100,0 & 100,0 & 100,0 \\
\hline Effectif & 102 & 184 & 286 & 292 & 238 & 530 \\
\hline
\end{tabular}

* : Les pourcentages sont obtenus après pondération

Source : Enquête sur les migrations, l'insertion urbaine et l'environnement au Burkina Faso 2000 (calculs de l’auteur)

\section{B) Lieu de départ des enfants confiés}

La circulation des enfants ne s'inscrit pas seulement dans une logique de départ d'enfants uniquement à partir du milieu rural. Hier comme aujourd'hui le lieu de départ est à la fois rural et urbain voire à l'étranger (tableau 2). Même si le milieu rural reste encore le lieu de départ prédominant, en passant de la génération la plus ancienne à la plus jeune, on note une diminution de cette importance au profit des autres villes mais, celle-ci est beaucoup plus marquée chez les garçons. Par ailleurs, pour la génération 
intermédiaire, les départs de la ville de Ouagadougou connaissent une augmentation relative indépendamment du sexe de l'enfant. Aussi bien la diminution de l'importance du milieu rural que celle de la croissance des départs à partir de Ouagadougou indique que la pratique de confiage au Burkina Faso subit aussi les effets de la crise économique.

Tableau 2: Répartition $(\%)^{*}$ des enfants confiés par zone de départ selon la génération et le sexe

\begin{tabular}{|l|cccc|}
\hline & \multicolumn{4}{|c|}{ Génération } \\
\hline Zone de départ & Avant 1980 & $1980-1989$ & $1990-1999$ & Total \\
\hline Garçon & & & & \\
Rural & 84,2 & 69,5 & 51,2 & 67,7 \\
Ouagadougou & 4,9 & 6,5 & 3,8 & 5,4 \\
Autres villes & 5,8 & 20,9 & 37,1 & 22,0 \\
Etranger & 5,1 & 3,1 & 7,8 & 4,9 \\
Total & $100(91)$ & $100(196)$ & $100(107)$ & $100(394)$ \\
Fille & & & & \\
Rural & 82,9 & 85,1 & 69,1 & 78,4 \\
Ouagadougou & 6,9 & 8,5 & 5,1 & 6,9 \\
Autres villes & 6,3 & 5,9 & 24,3 & 13,3 \\
Etranger & 3,9 & 0,4 & 1,5 & 1,3 \\
Total & $100(48)$ & $100(205)$ & $100(169)$ & $100(422)$ \\
Ensemble & \multicolumn{4}{|c}{} \\
Rural & 83,8 & 76,9 & 61,5 & 72,9 \\
Ouagadougou & 5,6 & 7,5 & 4,6 & 6,1 \\
Autres villes & 5,9 & 13,8 & 29,8 & 17,8 \\
Etranger & 4,7 & 1,9 & 4,2 & 3,2 \\
Total & $100(139)$ & $100(401)$ & $100(276)$ & $100(816)$ \\
\hline
\end{tabular}

* : Les pourcentages sont obtenus après pondération

( ): effectif

Source : Enquête sur les migrations, l'insertion urbaine et l'environnement au Burkina Faso 2000 (calculs de l'auteur)

\section{C) Lieu d'arrivée des enfants confiés}

Lorsqu'on passe de la plus vieille à la plus jeune génération, on observe une augmentation de l'importance du milieu rural et des autres villes et une diminution de celle de Ouagadougou, indépendamment du sexe de l'enfant confié (tableau 3). S'agissant du cas de Ouagadougou on note par exemple qu'en passant de la génération la plus ancienne à la plus récente la part des garçons accueillis dans cette ville diminue de façon drastique, passant de $33 \%$ à $1 \%$ et celle des filles passe de $17 \%$ à $6 \%$. La crise économique, certainement plus perceptible à Ouagadougou, déterminerait la réorientation des enfants vers d'autres destinations, particulièrement vers le milieu rural. Mais 
Zourkaléini Younoussi: Les Determinants Demographiques Et Socio-Economiques Du Confiage Des Enfants Au Burkina Faso

les résultats montrent que cette crise n'a pas entamé la capacité d'accueil des autres villes, les garçons sont de plus en plus accueillis et les filles de la génération la plus récente sont plus accueillies dans ces villes que l'ancienne génération.

Tableau 3: Répartition (\%)* des enfants confiés par zone de destination selon la génération et le sexe

\begin{tabular}{|l|cccc|}
\hline \multirow{2}{*}{ Zone de destination } & \multicolumn{4}{|c|}{ Génération } \\
\cline { 2 - 5 } Garçon & Avant 1980 & $1980-1989$ & $1990-1999$ & Total \\
Rural & 28,9 & 43,1 & 56,6 & 43,7 \\
Ouagadougou & 32,6 & 8,9 & 1,1 & 12,1 \\
Autres villes & 9,8 & 28,4 & 31,5 & 25,0 \\
Etranger & 28,7 & 19,6 & 10,9 & 19,2 \\
Total & $100(91)$ & $100(196)$ & $100(107)$ & $100(394)$ \\
Fille & & & & \\
Rural & 44,5 & 42,7 & 52,7 & 47,0 \\
Ouagadougou & 17,0 & 15,0 & 6,1 & 11,7 \\
Autres villes & 22,8 & 17,9 & 27,4 & 22,4 \\
Etranger & 15,7 & 24,4 & 13,8 & 19,0 \\
Total & $100(48)$ & $100(205)$ & $100(169)$ & $100(422)$ \\
Ensemble & & & & \\
Rural & 34,4 & 42,9 & 54,4 & 45,3 \\
Ouagadougou & 27,1 & 11,8 & 4,0 & 11,9 \\
Autres villes & 14,4 & 23,5 & 29,1 & 23,7 \\
Etranger & 24,1 & 21,9 & 12,5 & 19,1 \\
Total & $100(139)$ & $100(401)$ & $100(276)$ & $100(816)$ \\
\hline
\end{tabular}

* : Les pourcentages sont obtenus après pondération

( ): effectif

Source : Enquête sur les migrations, l'insertion urbaine et l'environnement au Burkina Faso 2000 (calculs de l'auteur)

D) Origine et destination des enfants confiés

Au tableau 4 on peut souligner une circulation des enfants à l'intérieur d'un même milieu et entre tous les milieux. La circulation Ouagadougou-zone rurale est devenue de plus en plus importante en passant de la génération la plus ancienne à la plus récente $(11,5 \%$ à $35,2 \%)$. Parallèlement, la circulation de la capitale vers les villes secondaires se poursuit. La détérioration des conditions de vie des ménages depuis la crise économique des années quatre-vingt a donc conduit certains parents résidant dans la capitale à envoyer leurs enfants au village ou vers les villes secondaires (Beauchemin 2001). 
On observe également une circulation des enfants à l'intérieur de la capitale. Le confiage à l'intérieur de la capitale a essentiellement pour but de rapprocher l'enfant de l'école ou de réduire les frais de scolarité (Baux et al. 2002).

En passant de la plus vieille à la plus jeune génération, le milieu rural prend une place importante de plus en plus dans la destination, indépendamment du lieu de provenance. Malgré la crise socioéconomique et peut-être aussi à cause d'elle, la place du milieu rural dans l'accueil des enfants de toute provenance, reste significative, ce qui traduit le fait que la solidarité familiale exprimé par le placement des enfants est encore vivace dans la société burkinabé.

Toutefois si la pratique du « confiage » des enfants reste bien ancrée dans la société, des fissures se créent et des manifestations d'exploitation des enfants sont observées. Les nouvelles formes prises par celle-ci permettent aux parents d'accueil de concilier à peu de frais, leurs rôles économique et éducatif $\mathrm{du}$ confiage tout en souscrivant aux principes d'obligations sociales qui conditionnent les relations familiales (Pilon 1996).

Tableau 4: Répartition (\%)* des enfants confiés par génération selon la zone de destination et de départ

Zone de départ

Zone de destination

$\begin{array}{lllll}\text { Rura } & \text { Ouagadougo } & \text { Autre } & \text { Etrange } & \text { Total } \\ \mathrm{l} & \mathrm{u} & \mathrm{s} & \mathrm{r} & \\ & & \text { villes } & & \\ & & & \end{array}$

Génération : Avant

1980

Rural

Ouagadougou

38,3

$29,0 \quad 9,9$

$22,9 \quad 100,0(68)$

11,3

$24,9 \quad 44,1$

$19,7 \quad 100,0(34)$

Autres villes

20,2

$20,9 \quad 44,7$

$14,3 \quad 100,0(25)$

Etranger

11,5

$3,2 \quad 21,8$

$63,5100,0(12)$

Total

$27,1 \quad 14,4$

$24,1 \quad 100,0(13$

Génération : 1980-1989

Rural

Ouagadougou

46,5

$12,0 \quad 16,5$

$25,1 \quad 100,0(22)$

27,7

$23,8 \quad 39,8$

$5,1 \quad 55,6$

$8,8 \quad 100,0(91)$

Autres villes

27,0

$5,5 \quad 8,9$

$12,4 \quad 100,0(76)$

Etranger

72,7

$11,8 \quad 23,5$

$12,9 \quad 100,0(12$

Total

42,9

$21,9 \quad 100,0(40$

Génération ; 1990-1999

Rural

56,4

$4,1 \quad 27,0$

$12,5 \quad 100,0(14$ 
Zourkaléini Younoussi: Les Determinants Demographiques Et Socio-Economiques Du Confiage Des Enfants Au Burkina Faso

$\begin{array}{lrrrrr}\text { Ouagadougou } & 35,2 & 27,5 & 26,4 & 10,9 & 100,0(61) \\ \text { Autres villes } & 51,8 & 0,6 & 34,9 & 12,7 & 100,0(60) \\ \text { Etranger } & 64,0 & 0,0 & 22,6 & 13,5 & 100,0(8) \\ \text { Total } & 54,4 & 4,0 & 29,1 & 12,5 & 100,0(27\end{array}$

* : Les pourcentages sont obtenus après pondération

( ): effectif

Source : Enquête sur les migrations, l'insertion urbaine et l'environnement au Burkina Faso 2000 (calculs de l'auteur)

\section{E) Rapidité d'accès au confiage}

L'accès au confiage est basé sur l'examen des probabilités cumulées, ou encore le risque de connaître un premier confiage au bout d'un âge inférieur ou égal à 13 ans. Le graphique 1 (courbe de Kaplan Meier) montre que la courbe d'accès au premier départ des filles reste toujours en dessous de celle des garçons. Cela porte à croire qu'elles rentrent plus rapidement en confiage que les garçons. Toutefois, un test de «Cox » nous permet de conclure que ces deux courbes ne sont pas significativement différentes. En somme, les filles ne se distinguent pas des garçons en termes de calendrier de l'accès au confiage.

Pour mesurer l'évolution du confiage dans le temps nous avons constitué des groupes homogènes par rapport au temps en scindant l'échantillon en cohortes ou en générations. Ainsi nous supposons que les individus qui les composent ont connu les mêmes conditions de vie, le même environnement, aux mêmes âges. Aujourd'hui, il semble que les enfants sont plus confiés qu'autrefois, filles comme garçons. En effet, au regard du graphique 2, on peut conclure que les probabilités d'être confiés augmentent en passant de la plus vieille à la plus jeune génération, quel que soit le sexe. L'hypothèse selon laquelle l'urbanisation et la crise économique (amoindrissant les solidarités familiales et les liens de réciprocités) devraient contribuer à faire diminuer cette pratique (Isiugo-Abanihe, 1994), n'est donc pas vérifiée par cette analyse. Bien au contraire, les enfants semblent être pris en compte dans les stratégies distributives de minimisation des risques liés à la crise économique et aux aléas climatiques. 


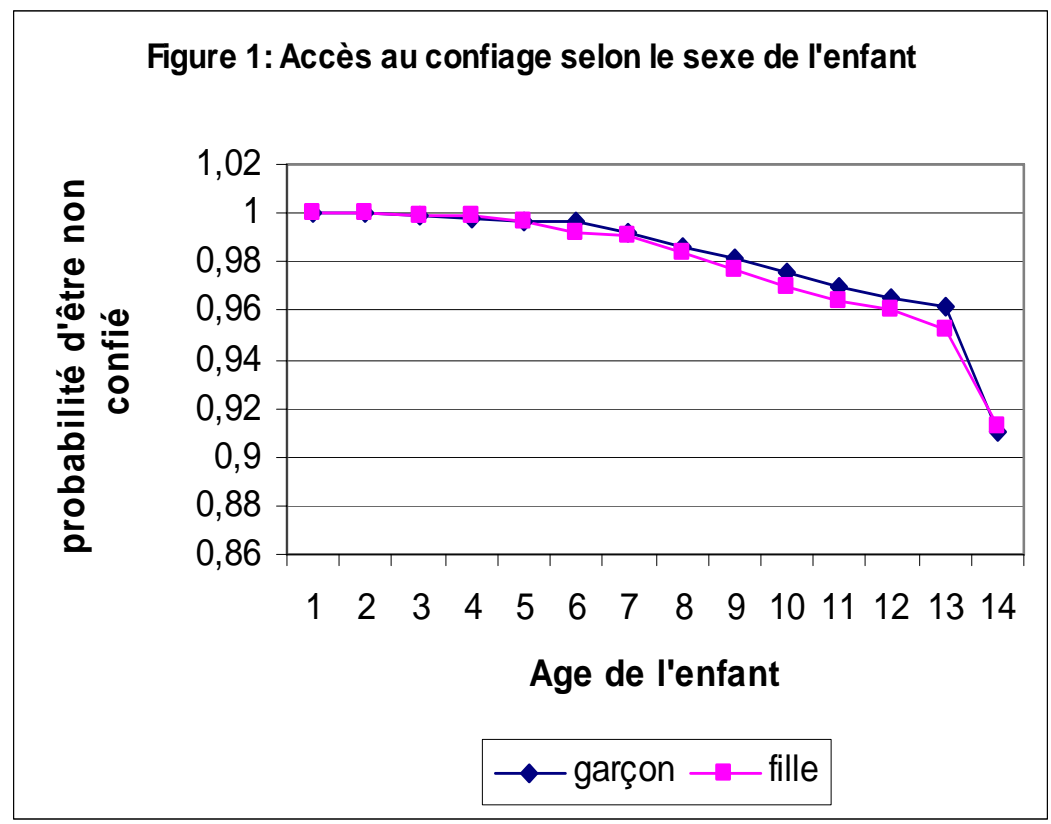

Source : Enquête sur les migrations, l'insertion urbaine et l'environnement au Burkina Faso 2000 (calculs de l'auteur)

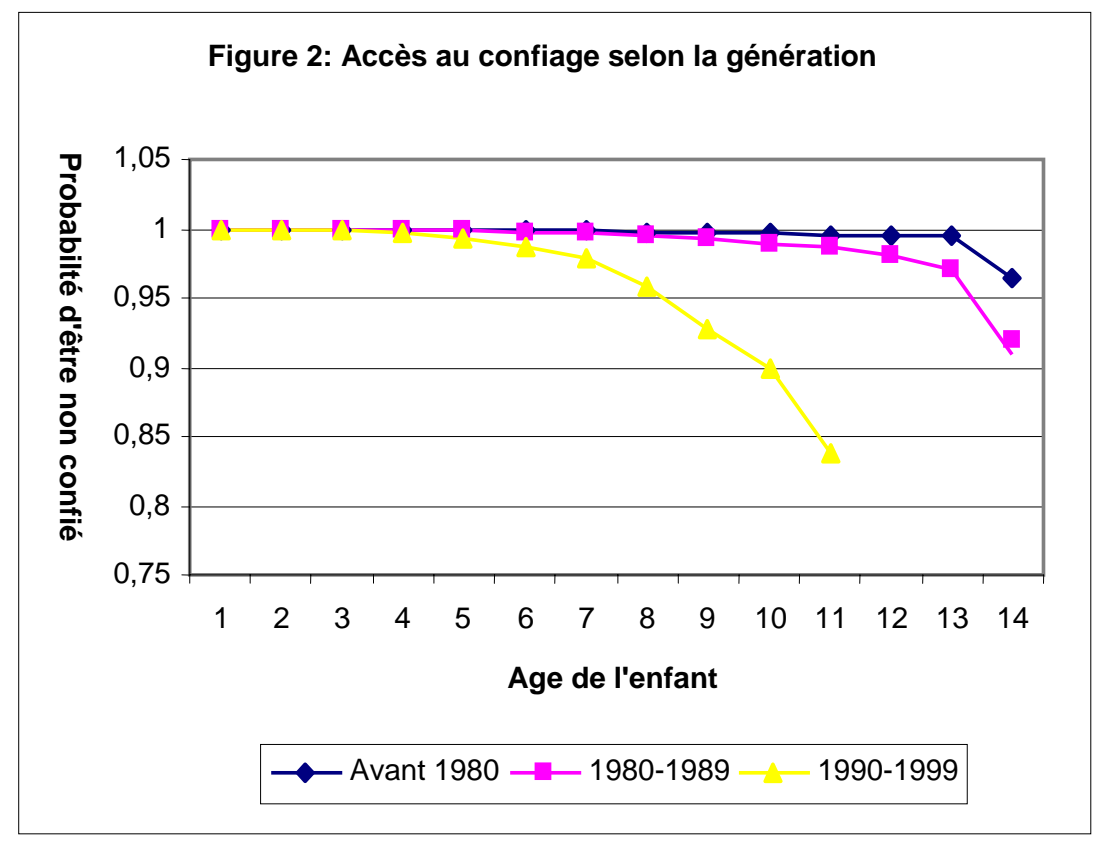

Source : Enquête sur les migrations, l'insertion urbaine et l'environnement au Burkina Faso 2000 (calculs de l'auteur) 
Zourkaléini Younoussi: Les Determinants Demographiques Et Socio-Economiques Du Confiage Des Enfants Au Burkina Faso

\section{IV) Déterminants du confiage des enfants}

Prenant en compte l'ensemble des enfants de toute la période couverte (colonne 2 tableau 5), il apparaît clairement une forte propension d'être confié en passant de la génération la plus ancienne à la plus jeune. Les difficultés économiques ont donc exacerbé la pratique de confiage des enfants. Très certainement, avec les diverses mutations des valeurs culturelles, les difficultés conjoncturelles, les aléas climatiques, cette augmentation de la pratique de confiage conduit à des dérapages qui aboutissent à un nouveau visage de ce phénomène social. A ce sujet, Fagnon et Kpadonou (1997, p. 1) font le commentaire suivant: "quand on parle d'enfant confié ou placé de nos jours, on ne perçoit plus vraiment ce sacrifice que s'imposaient autrefois les parents, de rester dans la logique des obligations traditionnelles et sociales, quel que soit leur rang socioéconomique.»

Désormais les effets de certaines valeurs et les perceptions traditionnelles relatives au confiage semblent relever du passé. En effet, si l'hypothèse selon laquelle les premiers enfants ont une probabilité plus élevée d'être placés semble être vérifiée en partie (à partir de 4 enfants) l'analyse par génération montre que cette moyenne d'ensemble n'est plus vérifiée de nos jours. En effet, après la génération née avant 1980, le rang de naissance ne semble plus avoir un effet significatif sur le confiage des enfants, toutes choses étant égales par ailleurs.

Pour les anciennes générations, le sexe de l'enfant n'est pas un déterminant significatif du confiage des enfants. Cependant on note un changement dans le temps de l'effet de cette variable. En effet, pour les deux premières générations les filles ne se distinguent pas significativement des garçons alors que pour la dernière génération elles semblent circuler plus. Le nouveau schéma vers lequel évolue la pratique de confiage des enfants semble donc privilégier l'accueil des filles aptes à être immédiatement employées comme aides familiales, indépendamment de leurs lieux de provenance ou de destination.

Un enfant qui compte beaucoup d'oncles et/ou tantes (plus de 20) semble être plus exposé à une mobilité entre familles. Cela appuie fortement l'hypothèse selon laquelle le confiage des enfants met en œuvre des réseaux de parenté et d'alliance, sources de solidarité susceptible de contribuer à l'amélioration de son bien 
être et de maintenir le mode de vie traditionnel au Burkina Faso. Mais des changements sont en train de s'opérer car si par le passé (la première génération) le simple fait d'avoir beaucoup $\mathrm{d}^{\prime}$ oncles et/ou de tantes a un effet propre sur le confiage des enfants, aujourd'hui (deux dernières générations) cette condition n'est plus suffisante pour favoriser la circulation des enfants entre familles.

L'ethnie Mossi, groupe majoritaire, est prise dans le cadre de cette étude comme la catégorie de référence. Certains groupes ethniques semblent avoir un comportement différent de celui des Mossi. Par exemple, lorsque la mère appartient au groupe Bobo ou Sénoufo, l'enfant à moins de chance d'être confié par rapport à celui dont la mère est de l'ethnie mossi. A l'opposé, l'enfant de mère Peul semble avoir plus de chance d'être confié que celui d'une mère Mossi. Ces résultats suggèrent donc que le mode de vie et les pratiques cultuelles propres à certaines ethnies influencent la circulation des enfants. Par exemple, la différence entre Peul et Mossi peut s'expliquer par le mode de vie des premiers, le nomadisme, qui ne leur permet pas de rester sur place durant toute une année scolaire les amène à placer les enfants auprès d'autres parents ou à l'internat pour assurer leur scolarisation ou l'apprentissage d'un métier lorsque des dispositions ne sont pas prises pour que l'école les accompagne dans ce déplacement. Néanmoins, l'analyse par génération ne confirme pas la constance de tels effets dans le temps. La faible représentativité de certains groupes ethniques dans l'échantillon en appel donc à des réserves sur la nature de ces résultats. Toutefois nous pouvons faire remarquer que ces résultats se rapprochent de ceux obtenus par Dabiré (2001), à partir d'une analyse des données de l'Enquête Démographique et de Santé réalisée en 1993.

La religion ne semble pas avoir un effet sur la pratique de confiage des enfants. Les résultats suggèrent que les conversions religieuses qui peuvent s'accompagner d'un changement des règles de parenté ou d'alliance et d'une conception différente de la personne n'entraînent pas un changement major en termes de placement d'enfants. Par exemple, en adoptant un mode vie proposé par la religion catholique, celui d'avoir un couple très solidaire, ayant pour fonction principale l'éducation des enfants et l'épanouissement affectif des partenaires, certaines familles n'arrivent pas à se soustraire des contraintes traditionnelles. 
Zourkaléini Younoussi: Les Determinants Demographiques Et Socio-Economiques Du Confiage Des Enfants Au Burkina Faso

Il ne semble pas y avoir une différence significative selon le niveau d'éducation de la mère. L'absence de différence significative suppose que les enfants circulent dans les mêmes proportions entre les deux types de familles (parents instruits et parents non instruits). Ainsi, même si un bon niveau d'éducation peut induire chez les adultes une aspiration à un style de vie plus proche de la famille nucléaire, les normes de l'entourage semblent plus ou moins s'y opposer. Souvent c'est l'aspiration à scolariser les enfants qui entraîne le confiage mutuel des enfants de frères et sœurs, indépendamment de leur niveau d'éducation. Cela dénote tout simplement la solidarité qui s'exerce à l'égard des membres d'une famille très étendue, de la campane et de la ville. Tous les individus peuvent avoir recours aux autres membres de leur famille, jusqu'à un niveau de parenté assez éloigné.

La situation dans l'activité de la mère est aussi un autre déterminant, surtout pour les générations anciennes: les mères salariées confient moins leurs enfants en comparaison à celles qui exercent une activité indépendante alors que les enfants de mères inactives ne se distinguent pas. Les enfants de celles disposant de revenu circulant moins on peut conclure que même si plusieurs raisons semblent justifier le placement, le confiage ou le transfert des enfants, la pauvreté reste la cause fondamentale. De nos jours on peut affirmer que dans cette situation de pauvreté généralisée aucune famille n'est épargnée et la situation dans l'activité ne fait désormais plus parti des facteurs explicatifs du confiage des enfants. Associée au confiage des deux premières générations, elle n'est plus pour la dernière génération: les enfants des femmes exerçant une activité indépendante ne se distinguent pas de ceux des femmes salariées ou inactives. Les solidarités très fortes qui s'imposent par l'éducation et les croyances, à tous les membres d'une famille, d'un lignage, font que, le confiage des enfants est utilisé comme stratégie de survie par l'ensemble des familles touchées.

Par ailleurs, même si la disponibilité d'oncles et tantes peut avoir un effet propre, c'est plus le lieu de résidence de ces derniers qui est plus déterminant. Le lieu de destination des enfants confiés concerne plus le milieu urbain ou un pays étranger. Par exemple un enfant qui quitte sa mère a sept fois plus de chances de se retrouver dans la capitale par rapport au milieu rural.

S'agissant du lieu départ, dans l'ensemble seule la ville de Ouagadougou se distingue du milieu rural avec une faible 
propension d'en partir. Mais l'analyse par génération inclut l'étranger comme un facteur qui émerge dans la structure des déterminants $\mathrm{du}$ confiage. Si pour la première génération Ouagadougou se distingue $\mathrm{du}$ milieu rural par la faible propension des enfants de la quitter pour un confiage pour les deux dernières générations il ne semble pas avoir de différence significative. A l'inverse, pour les enfants résidant à l'étranger c'est seulement pour la génération la plus récente qu'ils se distinguent significativement et fortement de ceux du milieu rural. Résidant à Ouagadougou, aujourd'hui les enfants urbains circulent autant que ceux du milieu rural. Avec la crise économique qui sévit dans les pays voisins, en particulier la Côte d'Ivoire qui accueille près de $90 \%$ des migrants Burkinabé vers l'extérieur, les enfants y quittent plus les centres urbains que le milieu rural. Ainsi, avec l'aggravation de la situation économique à Ouagadougou ou à l'étranger, de nombreux adultes contraints de prendre en charge de nombreux dépendants se retrouvent, $\mathrm{du}$ fait de la perte d'emploi, de la disparition de la clientèle potentielle de leur activité informelle, incapables de faire face à leurs obligations. Ils mettent donc à contribution les membres de leur famille encore au village ou ailleurs. Ceci confirme le fait que la solidarité entre membres d'une famille n'est pas en voie de disparition, contrairement à ce qu'on pourrait penser. Bien sûr, les jeunes " émancipés » qui demeurent apte à accueillir des enfants d'autres parents démunis laissent percer une certaine lassitude à devoir aider une parentèle multiple, mais bien peu oseraient se dérober à ces obligations (Locoh 1988).

Concernant le lieu d'arrivée, des changements se sont opérés également. La propension d'être accueilli à Ouagadougou diminue dans le temps en passant d'une différence fortement significative pour la première génération à une différence non significative pour la génération la plus récente. Pour la dernière génération les autres centres urbains accueillent moins d'enfants que le milieu rural. Devant l'accroissement dramatique des difficultés économiques en milieu urbain, ce sont les familles qui apportent les réponses les plus cohérentes. En l'absence de difficultés économiques les réseaux de solidarité entre familles et les normes d'assistance sont si vivaces que de nombreuses familles urbaines supportent des charges familiales diverses auxquelles elles ne peuvent se soustraire. Elles accueillent plus d'enfants que les familles rurales. Cependant devant la crise économique persistante on y envoie moins d'enfants chez des employés harcelés de soucis financiers, voire de dettes. Il n'est pas rare de voir une famille rurale prélever une portion non négligeable de ses 
Zourkaléini Younoussi: Les Determinants Demographiques Et Socio-Economiques Du Confiage Des Enfants Au Burkina Faso

maigres ressources pour envoyer de la nourriture à l'un de ses « dépendants » au chômage en ville, ou accueillir en son sein des enfants venus de la ville.

Tableau 5 : Déterminants démographiques et socio-économiques du confiage des enfants (regression de survie, modèle de cox)

\begin{tabular}{|c|c|c|c|c|}
\hline & & Générat & & \\
\hline & Ensemble & $\begin{array}{l}\text { Avant } \\
1980\end{array}$ & 1980-1989 & 1990-1999 \\
\hline Variable & $\begin{array}{l}\text { Hazard } \\
\text { ratios }\end{array}$ & \begin{tabular}{|l} 
Hazard \\
ratios
\end{tabular} & $\begin{array}{l}\text { Hazard } \\
\text { ratios }\end{array}$ & $\begin{array}{l}\text { Hazard } \\
\text { ratios }\end{array}$ \\
\hline Génération (1980-1989) & & & & \\
\hline Avant 1980 & $0,3 * * *$ & & & \\
\hline 1990-1999 & $14,4^{* * *}$ & & & \\
\hline Rang de naissance (premier) & & & & \\
\hline 2 & 1,0 & 1,1 & 1,1 & 1,0 \\
\hline 3 & 0,8 & 1,0 & 0,9 & 0,6 \\
\hline 4 et plus & $0,7 * * *$ & $0,7^{* *}$ & 0,9 & 0,6 \\
\hline Sexe (garçon) & & & & \\
\hline Fille & 1,0 & 0,7 & 0,9 & $1,4^{*}$ \\
\hline $\begin{array}{l}\text { Nombre d'oncle et/ou tantes } \\
\text { (moins de 10) }\end{array}$ & & & & \\
\hline 10 a 19 & 1,0 & $1,9 * *$ & 1,0 & 0,7 \\
\hline 20 et + & $1,4^{*}$ & $2,7^{* *}$ & 1,5 & 0,9 \\
\hline Ethnie de la mère (mossi) & & & & \\
\hline Bobo & $0,7 *$ & 1,1 & 1,0 & $0,3 * * *$ \\
\hline Peul & $1,8^{* *}$ & $3,5^{* *}$ & $2,0 *$ & 1,3 \\
\hline Dagara & $0,5^{* *}$ & $0,1^{* *}$ & 0,7 & 0,4 \\
\hline Senoufo & $0,7^{*}$ & 0,8 & 1,0 & $0,5^{* *}$ \\
\hline Gourounsi & 0,9 & 1,2 & 0,9 & 0,5 \\
\hline Bissa & 0,7 & $0,2 * * *$ & 0,6 & 1,2 \\
\hline Gourmantche & 0,8 & 1,0 & 0,9 & 0,7 \\
\hline Lobi & $0,6^{*}$ & $0,1^{* *}$ & 1,0 & $0,3^{* *}$ \\
\hline Autres ethnies & 1,1 & 0,9 & 1,2 & 1,0 \\
\hline Religion (musulmane) & & & & \\
\hline Animiste & 0,8 & 0,8 & 0,8 & 0,8 \\
\hline Catholique & 0,9 & 1,1 & 1,0 & 0,6 \\
\hline Protestante & 0,9 & 0,9 & 0,4 & 1,3 \\
\hline Education (aucun) & & & & \\
\hline Medersa & 1,3 & 0,7 & 0,9 & 2,0 \\
\hline Primaire & 1,0 & 1,3 & 0,9 & 1,1 \\
\hline $\begin{array}{l}\text { Secondaire et }+ \\
\text { Situation de la mère dans } \\
\text { l'activité (indépendante) }\end{array}$ & 1,6 & 1,0 & 1,4 & 1,8 \\
\hline Salarie & $0,3 * * *$ & $0,2^{*}$ & $0,3 * * *$ & 0,6 \\
\hline Apprenti/aide familial & 1,0 & 0,9 & 0,9 & 1,1 \\
\hline Inactif & 0,7 & 0,9 & 0,7 & 0,8 \\
\hline
\end{tabular}


African Population Studies Vol. $22 n^{\circ} 2$ /Etude de la population africaine vol. $22 N^{\circ} 2$

\begin{tabular}{|l|llll|} 
Lieu de départ (rural) & & & \\
Ouagadougou & $0,3^{* * *}$ & $0,2^{* * *}$ & 0,4 & 2,0 \\
Autres Urbains & 1,0 & 0,8 & 0,7 & 1,6 \\
Etranger & 0,9 & 0,8 & 0,6 & $2,8^{*}$ \\
Lieu de destination (rural) & & & & \\
Ouagadougou & $7,3^{* * *}$ & $12,7^{* * *}$ & $5,4^{* * *}$ & 0,5 \\
Autres Urbains & $2,7^{* * *}$ & $2,2^{*}$ & $3,0^{* * *}$ & 2,1 \\
Etranger & $3,6^{* * *}$ & $2,5^{* *}$ & $3,9 * * *$ & $4,1^{* * *}$ \\
\hline Nombre d'observations & 27821 & 7883 & 10059 & 9879 \\
Nombre d'enfants & 17121 & 4041 & 5522 & 7558 \\
Enfants confiés & 816 & 139 & 401 & 276 \\
\hline
\end{tabular}

$* * *: \mathrm{P}>|\mathrm{Z}|<=1 \% * *: \mathrm{P}>|\mathrm{Z}|<=5 \% \quad *: \mathrm{P}>|\mathrm{Z}|<=10 \% \quad(\quad)$ : catégorie de référence Source : Enquête sur les migrations, l'insertion urbaine et l'environnement au Burkina Faso 2000 (calculs de l'auteur)

\section{Conclusion et discussion}

La mise en évidence des différents facteurs de la circulation des enfants entre les familles nous a permis de constater que ce phénomène n'échappe pas à l'influence des inégalités démographiques et économiques entre les familles, des groupes sociaux et des milieux de résidence. Le rang de naissance, le nombre d'oncles et/ou de tantes apparaissent comme des facteurs favorisant la circulation des enfants dans le passé. Mais hier comme aujourd'hui, l'ethnie semble jouer un rôle important dans le confiage des enfants. Les enfants circulent entre le milieu rural et le milieu urbain et les femmes sans emploi sont plus portées vers cette pratique. A la question de savoir qu'elle signification donner à la pratique actuelle du confiage des enfants, tout porte à croire que le confiage des enfants demeure un mode d'organisation sociale et de gestion de la solidarité familiale. Mais ceci reste tributaire des contraintes environnementales. En effet, un enfant est envoyé dans un autre ménage que celui de ses parents biologiques, soit pour répondre à ses besoins en matière scolarisation ou de formation professionnelle, soit pour répondre à un besoin d'assistance du ménage d'accueil, soit pour soulager la famille d'origine. Toutefois, le recours à ces valeurs traditionnelles peut servir aujourd'hui de prétexte pour abuser des enfants. Cela pourrait peut-être justifier le fait qu'aujourd'hui les filles sont plus confiées que les garçons.

Cependant, hier comme aujourd'hui, les enfants sont l'avenir de la famille au sens large et tous les adultes sont concernés par leur prise en charge et leur formation. Ce principe est renforcé par la nécessité d'assurer l'adéquation ou tout au 
Zourkaléini Younoussi: Les Determinants Demographiques Et Socio-Economiques Du Confiage Des Enfants Au Burkina Faso

moins l'équilibre entre les membres actifs et les membres inactifs des ménages; ce qui conduit à une redistribution des enfants entre les unités familiales en particulier dans une conjoncture économique difficile (Antoine et Guillaume 1986). Hier la distribution était plus dirigée vers la capitale et aujourd'hui elle va dans toutes les directions (interne comme externe). L'intensification du confiage, à partir de tous les milieux de résidence et dans toutes les directions, confirme que ce phénomène dépend aujourd'hui davantage des changements observés dans les relations entre ville et campagne ainsi que dans les structures familiales, en réponse à la déstabilisation des familles pauvres actuelles (Vimard et Guillaume 1991).

L'effet puissant de la génération montre que l'évolution économique et sociale de ces dernières décennies n'a pas entamé l'intensité de ce comportement familial ; on peut même dire que la crise économique a conduit les familles les plus démunies à placer davantage leurs enfants, de manière temporaire ou de façon durable, dans des familles jouissant de conditions de vie moins précaires et/ou moins onéreuses. Les femmes sans revenu faisant plus recours à celles qui travaillent. Cependant les mutations économiques, démographiques et sociales du moment remettent profondément en question cet équilibre antérieur. La pauvreté se généralisant cette situation relève désormais du passé, travaillant ou non les enfants circulent autant. Certes le rang de naissance ou le nombre d'oncles et/ou de tantes ne semblent plus être un facteur explicatif important de ce phénomène de nos jours, mais comme le souligne Locoh (1995a), quand on mesure le rôle joué par les réseaux familiaux dans la prise en charge des faibles, des jeunes et des dépendants, dans la création d'emplois, dans l'innovation de normes culturelles, pour ne citer que quelques secteurs clés, on est plus enclin à admirer leur vitalité qu'à atermoyer sur la perte des valeurs traditionnelles. Ce sont plutôt les caractéristiques socio-économiques du milieu de résidence de ce réseau de parenté qui captent certainement les effets de ces variables.

Cependant les solidarités traditionnelles, extraordinairement opérantes jusqu'à aujourd'hui, semblent atteindre leurs limites. En effet, les transferts d'enfants s'intègrent dans un système général de droits et d'obligations. Mais au Burkina Faso comme ailleurs en Afrique, elles sont ébranlées en cette période de déflation prolongée et d'ajustement structurel qui caractérise aujourd'hui les économies africaines (Mahieu 1989). Ce 
système présente des limites puisque de plus en plus de ménages vivent à la marge (les charges induites par leurs obligations dépassent leurs revenus salariaux) et doivent développer des pratiques de crise qui restreignent les possibilités d'accueil des individus n'appartenant pas à la famille restreinte (Vidal et Le Pape, 1986). Les conséquences sur les solidarités familiales se font très fortement sentir : enfants de la rue, femmes abandonnées avec ou sans leurs enfants, parents âgés ou malades privés du soutien de leurs descendants, déscolarisation des enfants du fait du chômage des parents, sont aujourd'hui monnaie courante.

Dans ce contexte, il arrive de nos jours que les aspirations des parents et des enfants soient contradictoires. Les ménages d'accueil peuvent chercher à concilier à peu de frais, leurs rôles économique et éducatif tout en souscrivant aux principes d'obligation sociale qui conditionnent les relations familiales (Pilon 1996). Ainsi, l'enfant est placé pour travailler et gagner de l'argent et ce sont les parents et/ou les placeurs qui bénéficient des fruits de ses efforts, si ce n'est de sa servitude. Aussi, il semble que les filles sont plus recherchées pour ce genre de placement en ville où elles se consacrent aux activités domestiques et commerciales (Poirier et al. 1996).

Il convient de mettre sur pied des dispositifs d'enquête et d'observation plus précis pour identifier clairement la nature des travaux imposés à l'enfant, le degré de contrainte et d'exploitation qui existe entre les parents qui confient leurs enfants et ceux qui les accueillent pour les faire travailler. Tout porte à croire qu'il existe aujourd'hui des déviations par rapport aux normes d'expression de solidarité familiale. Il est donc important de répondre aux questions de savoir si le placement n'est pas devenu une activité mercantile et un mode d'exploitation du travail rémunéré des enfants, avec l'accord plus ou moins implicite des parents. Les parents ne placent-ils pas un enfant dans le but d'obtenir une rémunération, pouvant les aider à faire face aux charges des enfants restés à la maison ? Le placement n'est-il pas devenu une activité mercantile et un mode d'exploitation du travail rémunéré des enfants, avec l'accord implicite des parents, en réponse à une demande de plus en plus forte de main d'œuvre faiblement rémunérée ou gratuite en ville ? Après tout, un enfant placé auprès d'une autre famille pour des motifs économiques n'est-il pas soumit à des frustrations, des blessures narcissiques au sein de la famille d'accueil ? En effet, les enfants confiés peuvent se sentir diminués par rapport à leurs homologues biologiques, surtout quand on exhibe chaque fois les conditions de vie 
Zourkaléini Younoussi: Les Determinants Demographiques Et Socio-Economiques Du Confiage Des Enfants Au Burkina Faso

misérables de leur famille d'origine. L'amélioration des conditions de vie des enfants passe par des programmes de lutte contre la pauvreté qui tiennent compte à la fois de ses dimensions monétaires et non monétaires.

\section{BIBLIOGRAPHIE}

Antoine Ph ., Guillaume A. 1986. «Une expression de solidarité familiale à Abidjan : enfants du couple et enfants confiés ». In les familles d'aujourd'hui, 289-297, INED, Paris.

Baux Stéphanie, Lokpo K, Pilon M. 2002. "Confiage et scolarisation : une relation ambivalente » Ouaga Focus, n 10.

Beauchemin C. 2001. L'émergence de l'émigration urbaine en Côte d'Ivoire, radioscopie d'une enquête démographique (19881993), les études du CEPED n ${ }^{\circ} 19$, Paris.

Dabiré H. B. 2001. « Le confiage des enfants, alternative à une transition de crise ». In Les transitions démographiques des pays du Sud sous la direction de Gendreau F. et Poupard M., 407-421, AUPELF/UREF, Paris.

Fagnon P. et Kpadonou E. 1997. Phénomène des enfants confiés (Vidomegon) au Bénin, ampleur, conséquences, interventions, perspectives. Université du Bénin, Institut national d’économie, centre de formation et de recherche en matière de population, Cotonou.

Frank O. 1985. «La mobilité des enfants et l'autosuffisance économique des femmes dans le milieu patriarcal africain ». In Femmes et Politiques Alimentaires, 641-652. ORSTOM, Collection Colloques et Séminaires, Paris.

Institut National de la Statistique et de la Démographie (INSD) et ORC Macro. 1994. Enquête démographique et de Santé 1993. Calverton Maryland, USA : INSD et ORC Macro.

Institut National de la Statistique et de la Démographie (INSD) et ORC Macro. 1999. Enquête démographique et de Santé 1998. Calverton Maryland, USA : INSD et ORC Macro. 
Institut National de la Statistique et de la Démographie (INSD) et ORC Macro. 2004. Enquête démographique et de Santé 2003. Calverton Maryland, USA : INSD et ORC Macro.

Isiugo-Abanihe C. 1985. "Child fosterage in West Africa", Population and development review, vol 11, number 1, pp. 53-73.

Jonckers D. 1994. « Adoption et alliance chez les Minyanka Banama du Mali ». In Droits et Cultures, nº 27, 105-134.

Jonckers D. 1997. « Les enfants confiés ", in Ménages et Familles en Afrique, Approches des Dynamiques Contemporaines, sous la direction de Pilon M.., 193-208, Les études du CEPED n 15, Paris.

Lallemand S. 1993. La circulation des enfants en société traditionnelle, prêt, dons, échange L’Harmattan, Paris.

Lallemand S. 1994. Adoption et Mariage, les Kotokoli du Centre du Togo. L’Harmattan, Paris.

Lloyd C. et Blanc K. 1996. "Children’s schooling in Sub-Saharan Africa. The role of fathers, mothers, and others" Population and development review, vol 22, number 2, pp. 265-298.

Locoh T. 1988. "Structures Familiales et changements sociaux ». In Population et Société en Afrique au sud du Sahara, sous la direction de Tabutin D., 441-478. L'Harmattan, Paris.

Locoh T. 1995a. Familles africaines, Population et qualité de vie. Les dossiers du CEPED n ${ }^{\circ}$ 1, Paris.

Locoh T. 1995b. "Structures Familiales d'accueil des migrants et développement des structures familiales multipolaires en Afrique ». In Migration, Changements sociaux et développement sous la direction de Quesnel A. et Vimard P., 279-295, ORSTOM, Collection Colloques et Séminaires, Paris.

Mahfoud D. 1994. "Changement social et solidarité familiale: problématique, méthodologie et éclairage théorique ». In l'Entraide Familiale, 11-24, IREP, cahier 11, Tunis.

Mahieu F. R. 1989. "Transferts et Communauté africaine", STATECO, n58-59, 107-135. 
Zourkaléini Younoussi: Les Determinants Demographiques Et Socio-Economiques Du Confiage Des Enfants Au Burkina Faso

Marcoux R. 1997. «Le travail, un jeu d'enfant? A propos de la contribution des enfants à la subsistance des ménages au Mali ». In Ménages et Familles en Afrique, Approches des Dynamiques Contemporaines, 193-208. Les études du CEPED n ${ }^{\circ} 15$

Pilon M. 1996. " Les familles africaines en plein remueménage «. La chronique du CEPED, n²1.

Poirier J., Baya B., Zoungrana C. 1996. "Travail des enfants et division des tâches au sein des ménages ». Les travaux de $l^{\prime}$ UERD, n² , Ouagadougou.

Vidal A. et Le Pape M 1986. Pratiques de crise et conditions sociales des enfants à Abidjan, ORSTOM-CNRS, Paris.

Vimard P. et Guillaume A. 1991. "Mobilités Familiales et spatiales des enfants en Côte d'Ivoire ». In Migration, Changements sociaux et développement sous la direction de Quesnel A. et Vimard P., 243-260, ORSTOM, Collection Colloques et Séminaires, Paris. 